\title{
ARTICLE Chronic non-discriminatory social defeat is an effective chronic stress paradigm for both male and female mice
}

\author{
Christine N. Yohn ${ }^{1}$, Andrew Dieterich ${ }^{1,2}$, Allyson S. Bazer ${ }^{1}$, Isabella Maita ${ }^{1,2}$, Megan Giedraitis ${ }^{1}$ and Benjamin Adam Samuels ${ }^{1,2}$
}

\begin{abstract}
Stress-related mood disorders are more prevalent in females than males, yet preclinical chronic stress paradigms were developed in male rodents and are less effective in female rodents. Here we characterize a novel chronic non-discriminatory social defeat stress (CNSDS) paradigm that results in comparable stress effects in both sexes. Male and female C57BL/6J mice were simultaneously introduced into the home cage of resident CD-1 aggressors for 10 daily 5-min sessions. CD-1 aggressors attacked males and females indiscriminately, resulting in stress resilient and susceptible subpopulations in both sexes. CD-1 aggressors attacked C57BL/ 6J male intruders faster and more frequently than female intruders. However, CNSDS similarly induced negative valence behaviors in SUS mice of both sexes relative to RES and CNTRL mice. Furthermore, SUS male and female mice displayed similar increases in plasma corticosterone levels following CNSDS exposure relative to pre-stress exposure levels. The estrous cycle did not impact CD-1 attack behavior or negative valence behaviors. Thus, CNSDS induces chronic stress behavioral and neuroendocrine effects in both male and female C57BL/6J mice and allows direct comparisons between sexes. Adoption of this modified social defeat paradigm will help advance the initiative to include female rodents in preclinical chronic stress research.
\end{abstract}

Neuropsychopharmacology (2019) 44:2220-2229; https://doi.org/10.1038/s41386-019-0520-7

\section{INTRODUCTION}

Stress-associated psychiatric disorders, such as anxiety and depression, occur twice as frequently in women than men [1]. However, preclinical research investigating stress effects often excludes female subjects [2], possibly because female brain and behavior is impacted by fluctuations in gonadal hormones across the estrous cycle [3-5]. However, the estrous cycle does not result in more variability in female behavioral, molecular, and physiological traits than what is observed in male rodents [6-8]. Sex differences in stress response are prevalent at all levels of biological organization, yet the majority of chronic stress paradigms used in rodents were designed for and are only effective in males [9-16]. Investigating the validity and reliability of chronic stress paradigms to produce stress susceptibility phenotypes in both sexes is necessary for understanding the etiology of stress-associated mood disorders.

One such chronic stress paradigm, chronic social defeat stress (CSDS), exposes experimental male mice to 10 daily residentintruder bouts with an aggressor CD-1 male and results in both susceptible (SUS) mice, which display behavioral, neural, and hormonal alterations consistent with chronic stress, and resilient (RES) mice, which do not show these alterations [9, 10, 14, 16-19]. However, when female mice are exposed to CSDS, CD-1 male aggressors display mounting behaviors rather than aggression toward female intruders [20]. Female resident aggressors do not produce the necessary bouts of attack characterizing CSDS, as female-female aggression is limited to territorial species such as Peromyscus californicus [15] and prairie voles [21], maternal aggression [22, 23], or following ovariectomy [20]. To circumvent this limitation, Takahashi and colleagues [24] demonstrated that chemogenetic activation of the ventral medial hypothalamus in CD-1 mice induces aggression toward female intruders and subsequent social avoidance behaviors in the females. Furthermore, Harris and colleagues [25] showed that application of male CD-1 urine to $\mathrm{C} 57 \mathrm{BL} / 6 \mathrm{~J}$ female intruders induces attacks by resident male aggressors, resulting in social avoidance and decreased sucrose preference behaviors in the females. These methods, while clever and useful, require either difficult and time-consuming surgeries that target a deep structure or tedious urine collection.

Here we developed a chronic non-discriminatory social defeat stress (CNSDS) paradigm that is effective in both male and female C57BL/6J mice. We demonstrate that simultaneous exposure of both male and female sexually inexperienced intruder C57BL/6J mice to an aggressive resident CD-1 mouse yields aggression towards both C57BL/6J mice. Over the course of the 10-day CNSDS paradigm, the CD-1 repeatedly attacked both the male and female mice. These attacks resulted in behavioral and neuroendocrine responses in both sexes consistent with chronic stress exposure. This streamlined novel non-discriminatory social defeat paradigm will greatly facilitate the inclusion of female rodents in chronic stress research and permit direct comparisons between males and females.

\section{METHODS}

Subjects

Eight-week-old female and male C57BL/6J strain mice were purchased from Jackson Laboratories (Bar Harbor, ME). All mice were maintained on a 12L:12D schedule with lights coming on at 6 a.m. and going off at 6 p.m., with food and water provided ad libitum. All behavioral testing took place in the morning, between

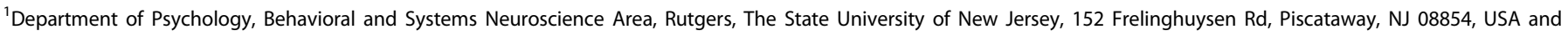
${ }^{2}$ Graduate Program in Neuroscience, Rutgers, The State University of New Jersey, New Brunswick, Piscataway, NJ 08854, USA
}

Correspondence: Christine N. Yohn (cy253@scarletmail.rutgers.edu) or Benjamin Adam Samuels (ben.samuels@rutgers.edu)

Received: 22 May 2019 Revised: 8 August 2019 Accepted: 29 August 2019

Published online: 7 September 2019 
the hours of 8 a.m. and 11 a.m. All experiments were conducted in compliance with $\mathrm{NIH}$ laboratory animal care guidelines and approved by Rutgers University Institutional Animal Care and Use Committee.

\section{Chronic non-discriminatory social defeat stress}

To determine if female mice experience a similar stress outcome as the standard CSDS paradigm in males [26], both female and male mice were exposed to a novel, dual-sex stress protocol. Adult male and female C57BL/6J mice were randomly assigned to either chronic stress or no stress control groups. Retired CD-1 breeder adult males were purchased from Charles River Laboratory (Wilmington, MA). To screen for aggressive behavior, screener female and male C57BL/6J mice were simultaneously placed in the home cage of a CD-1 mouse. CD-1 mice that reliably attacked both female and male screener mice within $60 \mathrm{sec}$ on consecutive days were selected as aggressors [27]. Two CD-1 mice were selected for each pair of experimental mice (one male and one female C57BL/ $6 \mathrm{~J}$ mouse). During the experimental phase, experimental male C57BL/6J mice were matched with the same experimental female C57BL/6J mice throughout the duration of the social defeat. The male and female $\mathrm{C} 57 \mathrm{BL} / 6 \mathrm{~J}$ pairs were placed into the home cage of novel CD-1 aggressors for 10 consecutive daily 5-min sessions. Since our cages only permitted one cage divider, we did not cohouse the aggressor CD-1 with both the experimental male and female $\mathrm{C} 57 \mathrm{BL} / 6 \mathrm{~J}$ mice. Instead, on alternating days, either the male or female C57BL/6J mice were housed with either the CD-1 aggressor mouse with which they had interacted or a novel control aggressor CD-1 (previously screened for aggressive behavior). Co-housed subjects were separated by clear, perforated plexiglass that permitted sensory but not physical interaction. In total, both C57BL/6J male and female mice were exposed to 5 days of sensory exposure to the aggressor CD-1 and 5 days exposure to a novel aggressor CD-1 (previously screened for aggressive behavior). A separate group of female and male C57BL/ 6J mice were simultaneously placed into a standard cage and allowed to interact for $5 \mathrm{~min}$ in the absence of an aggressor CD-1 male over 10 days. These opposite sex control mice were housed on either side of a divider and placed on a separate rack from stress mice for $24 \mathrm{~h}$ until the next interaction session.

To determine if the number of mice interacting affected control group behavior, we ran an additional cohort of controls that had three mice interacting (two males and one female; 2-male CNTRL) and compared the results to the original control group that had two mice interacting (one male and one female; 1-male CNTRL). The 2-male CNTRL group consisted of three mice interacting for 5 min per day for 10 consecutive days. Following the daily 5 -min interaction, male experimental mice were housed, separated by a divider, with the C57 male they just interacted with. The female experimental mice were then housed with an unfamiliar C57BL/6J male, separated by clear, perforated plexiglass.

Videos were recorded (Sony HDR-CX260) to measure attack latency and frequency, and mounting behavior. CD-1 mounting behavior toward female mice occurred in $8 \%$ of the interactions, with an average attack latency of 121s for RES and 150s for SUS female mice. In males, the CD-1 mice displayed mounting behaviors in $3 \%$ of the interactions, with an average attack latency of $87 \mathrm{~s}$ for RES and $76 \mathrm{~s}$ for SUS male mice. No mounting by the CD-1 led to pregnancy in any of the C57BL/6J females throughout the entire course of the experiment. In total 30 male and 30 female C57BL/6J mice were exposed to the CNSDS paradigm, which resulted in RES (male $=13$; female $=11$ ) and SUS (male $=17$; female $=19$ ) phenotypes. Additionally, 15 male and 15 female C57BL/6J mice were assigned to the CNTRL group.

\section{Vaginal lavage}

To assess estrous cycle state, daily vaginal lavages were performed 2 weeks prior to stress exposure to ensure mice were cycling throughout all four stages regularly. Subsequent to daily 5-min aggressive bouts and after completing each behavioral test or blood collection, vaginal smears were collected via a pipette filled with $\mathrm{ddH}_{2} \mathrm{O}$, placed at the opening of the mouse's vaginal canal (without penetration) with $\mathrm{ddH}_{2} \mathrm{O}$ gently expelled and suctioned back into the pipette tip [28, 29]. Samples were imaged under an EVOS FL Auto 2.0 microscope (Thermo Fisher Scientific, Waltham, MA) at 10x magnification. Estrous phases were identified by the presence or absence of nucleated epithelial cells, cornified epithelial cells, and leukocytes [28, 30]. Proestrus mice had mostly nucleated and some cornified cells, estrus predominantly displayed cornified epithelial cells, metestrus had cornified epithelial cells and polymorphonuclear leukocytes [28], while diestrus contained polymorphonuclear leukocytes. No differences in length of estrous cycle was observed 10 days before stress or the 10 days during stress between the CNTRL and CNSDS mice (Supplemental Fig. 3A). Additionally, RES and SUS mice had similar cycle lengths both during the 10 days before stress and 10 days after stress (Supplemental Fig. 3B).

\section{Behavioral testing}

Social interaction test. To assess responsiveness to CNSDS, we ran the social interaction test (SIT) to analyze social interaction and avoidance behavior in the presence of a novel CD-1 mouse. Mice were placed in an open field (OF) arena for two consecutive 2.5-min trials. The first trial had the CD-1 absent, with the CD-1 present in a perforated Plexiglas container within the social interaction zone $(14 \mathrm{~cm} \times 24 \mathrm{~cm})$ during the second trial [26, 27]. Overhead cameras recorded behavior, and EthoVision software (Noldus, Wageningen, Netherlands) measured time spent in the interaction zone. Social avoidant and interaction behavior were measured by time spent in the interaction zone during the first (CD-1 absent) and second (CD-1 present) trials, with an interaction ratio calculated: ((interaction time, CD-1 absent)/(interaction time, CD-1 present)).

Light dark. Within OF arenas, dark plastic rectangular boxes (opaque to visible light, but transparent to infrared light) covering $1 / 3$ of the arena, were inserted to separate the arena into light and dark compartments. The dark box contained an opening that allowed passage between the light and dark [11], with the light compartment brightly illuminated (1000 lux; Kinder Scientific). At the beginning of each 5-min test, mice were placed in the dark compartment, with distance traveled in the light compartment ((distance traveled in the light compartment/total distance) $\times 100$ ) and time in the light compartment used for analyses.

Elevated plus maze. The elevated plus maze (EPM) test consisted of a plus-shaped apparatus with two open and two closed arms (side walls), elevated 2 feet above the floor. During the 5-min test, the mice were recorded from a video camera mounted on the ceiling above each EPM arena. EthoVision software (Noldus, Wageningen, Netherlands) was used to quantify distance traveled in the open arms ((total open arm distance/total distance traveled) $\times 100$ ), and time on open arms used for analyses. EthoVision detected when 2 paws crossed a transition.

Novelty suppressed feeding. Mice were food deprived for $18 \mathrm{~h}$ in their home cage prior to being placed in the corner of a novel, brightly-lit (1500 lux) testing apparatus $(50 \times 50 \times 20 \mathrm{~cm})$ filled with $2 \mathrm{~cm}$ of corncob bedding, and a single food pellet attached to a white platform in the center. The novelty suppressed feeding (NSF) lasted $6 \mathrm{~min}$ with latency to eat (defined as the mouse sitting on its haunches and biting the pellet with the use of forepaws) recorded. Mice that timed-out were assigned a latency of 360 secs. Immediately after, mice were transferred to their home cages and given ad libitum access to a food pellet for $5 \mathrm{~min}$. Latency to eat and amount of food consumed was measured as a control for feeding behavior observed in the NSF. 
Sucrose preference test. Mice were habituated to a $1 \%$ sucrose solution in their home cage for 2 days. Next, a bottle containing water and a bottle containing $1 \%$ sucrose were placed side-byside and switched every $12 \mathrm{~h}$ for 3 days. Consumption of both water and sucrose bottles were measured and compared to determine preference for a $1 \%$ sucrose solution. Preference was calculated as $1 \%$ sucrose consumed divided by total consumption.

Blood collection and corticosterone ELISA

Mice were weighed to ensure that non-terminal blood collection was no more than $1 \%$ of the mouse's body weight. Prior to stress, baseline blood samples were collected from the left retro-orbital sinus of all experimental mice, in accordance with IACUC guidelines. To measure corticosterone levels in response to CNSDS, blood was collected from the right retro-orbital sinus 40-45 min after the 10th day of CNSDS. CNTRL mice had blood collected from the right retro-orbital sinus $40-45 \mathrm{~min}$ after the 10th day of opposite sex exposure. For each blood collections, blood was collected in microcentrifuge tubes coated with EDTA. Plasma was isolated from whole blood by centrifugation at 14,000 rpm for 10 min at $4{ }^{\circ} \mathrm{C}$, with supernatant collected and stored at $-80^{\circ} \mathrm{C}$ until assayed. Total yield of plasma per blood collection was between 25 and $40 \mu \mathrm{L}$. To assess differences in plasma corticosterone in response to CNSDS, baseline levels were compared to samples after CNSDS) across 6 mice per sex per stress susceptibility phenotype (male: $\mathrm{CNTRL}=6, \mathrm{SUS}=6$, RES $=$ 6; female: $C N T R L=6$, SUS $=6$, RES $=6$ ). Each sample (total $n=72$ across all time-points) was diluted 1:100 and assayed in triplicate according to the manufacturer's protocol (Arbor Assays Corticosterone ELISA Kit).

\section{Statistical analyses}

To investigate sex differences in CD-1 attack behavior (attack frequency and attack latency) independent samples $t$-tests were used. Separate $X^{2}$ analyses were used to assess if frequency of attack behavior was different between sex and estrous cycle phases. To assess sex and stress susceptibility effects $2 \times 3$ analysis of variance (ANOVA) with Bonferroni post-hoc comparisons were used to analyze behavioral results from SIT, LD, EPM, Sucrose preference, and Emotionality Index. Prior to conducting each ANOVA, we ran a Sharpio-Wilk normality test. Each ANOVA reported in the manuscript passed the Sharpio-Wilk ( $p$-values > $0.05)$, which justified usage of parametric analyses. When there was no main effect of sex in the ANOVAs, the mice were collapsed and Bonferroni post-hoc comparisons were used to compare stress susceptibility phenotype groups. Planned Bonferroni posthoc comparisons (based on the collapsed mice) were then used to investigate effects within each sex. To investigate differences in stress susceptibility across the estrous cycle $3 \times 4$ ANOVAs were used for all behaviors except NSF. Since NSF data fails to meet basic assumptions of normality, Kaplan-Meier survival analysis (nonparametric test) was used. Lastly, to analyze differences in endogeneous corticosterone levels, time point of blood collection, sex, and stress susceptibility were used to conduct a $3 \times 2 \times 2$ repeated measures ANOVA. Bonferroni post-hocs in collapsed mice and planned comparisons within each sex were then performed as was done for behavior analyses. GraphPad Prism 7 was used for all analyses.

\section{RESULTS}

CNSDS induces susceptible and resilient stress susceptibility phenotypes and negative valence behaviors in both males and females

To determine whether simultaneous exposure of $\mathrm{C} 57 \mathrm{BL} / 6 \mathrm{~J}$ male and female intruder mice to resident CD-1 aggressors results in attacks of both sexes, we modified a standard CSDS protocol. To this end, C57BL/6J male $(n=30)$ and female $(n=30)$ mice were exposed simultaneously to male CD-1 aggressor mice for 10 daily 5 -min aggressive bouts. Non-stressed control (CNTRL) C57BL/6J male $(n=15)$ and female $(n=15)$ mice experienced 5-min daily interactions with each other and were then co-housed (Fig. 1a). Independent samples $t$-tests revealed sex differences in CD-1 attack latency $(t(28)=3.85, p=0.006)$ and number of attacks $(t(28)=3.7, p=0.009$; Fig. 1b), with males on average being attacked more quickly and frequently across the 10 days than females. We next analyzed attack and mounting behaviors from resident CD-1 males on both male $(n=30)$ and female $(n=30)$ C57BL/6J intruder mice $(n=60)$. Male C57BL/6J mice were attacked in a total of $83.77 \%$ and were mounted in a total of $2.6 \%$ of their interactions with CD-1 aggressors (Fig. 1c). $14.94 \%$ of resident-intruder interactions resulted in neither aggression nor mounting of the male C57BL/6J mice (Fig. 1c). Female C57BL/6J mice were attacked in a total of $64.33 \%$, mounted in $7.64 \%$, and neither in $31.21 \%$ of these interactions (Fig. 1c). A $X^{2}$ test revealed a significant sex difference $\left(X^{2}=15.75, p<0.0001\right)$, with males being attacked more frequently than females. Estrous cycle stage in females did not impact resident-intruder aggression by the CD-1 aggressor $\left(X^{2}=9.03, p=0.434\right.$; Fig. $\left.1 \mathrm{C}\right)$. In addition, $71.34 \%$ of the CD-1 aggressors attacked both males and females (Fig. 1d), while just 16.56 and $9.55 \%$ of CD-1 aggressors attacked males or females only, respectively. Taken together, these data demonstrate that in most CNSDS interactions CD-1 aggressors will attack both the male and female C57BL/6J mice.

Similar to classic CSDS paradigms, we next used a social interaction test (SIT) to assess stress susceptibility in a new cohort of CNSDS and CNTRL male and female C57BL/6J mice (Fig. 2a, b). An interaction ratio ((interaction time with aggressor present)/ (interaction time with aggressor absent)) score of $<1$ resulted in a susceptible (SUS) classification, whereas resilient (RES) mice had an interaction ratio $>1[18,31]$. A $2 \times 3$ ANOVA (sex $\times$ stress susceptibility) was used to analyze stress susceptibility phenotype and sex differences in SIT behavior between SUS, RES, and CNTRL mice. This $2 \times 3$ ANOVA revealed no main effect of sex within SIT $(p=0.38)$. By contrast, a main effect of stress $(F(2,83)=23.21, p<$ 0.001 ) was observed. Therefore, given that no sex differences were observed, we first collapsed all mice and ran Bonferroni post-hoc comparisons (Fig. 2b left), which showed that SUS mice had a lower interaction ratio than RES $(p<0.0001)$ and CNTRL $(p=$ 0.0003 ; Fig. $2 \mathrm{~b}$ left) mice. We next ran planned Bonferroni posthoc comparisons (based on the collapsed comparisons) in both males and females. These separate planned Bonferroni post-hoc comparisons within sex showed that SUS males had a lower interaction ratio than RES $(p<0.001)$ and CNTRL $(p=0.036)$ males (Fig. 2b right) and that SUS females had a lower interaction ratio than RES $(p<0.001)$ and CNTRL $(p=0.005)$ females (Fig. 2b right).

Similar to our observations in Fig. 1, $2 \times 2$ ANOVAs (sex $\times$ stress susceptibility) revealed an effect of sex in average attack latency $(F(1,26)=16.99, p=0.0003$; Supplemental Fig. $1 \mathrm{~A}$ small panel $)$ and total number of attacks $(F(1,26)=14.65, p=0.0007$; Supplemental Fig. 1B small panel) by CD-1 aggressors across the 10 days, with SUS males on average attacked more quickly $(p=0.0017$; Supplemental Fig. 1A large panel) and more frequently $(p=$ 0.0053; Supplemental Fig. 1B large panel) than SUS females. Nevertheless, similar to other findings in classic CSDS paradigms [18], no main effect of stress susceptibility phenotype on attack latency $(p=0.51)$ or number of attacks $(p=0.15)$ across the 10 days existed between SUS and RES mice within males and within females (Supplemental Fig. 1A, B large panel).

We next subjected the CNSDS and CNTRL mice to behavioral tests that are affected by classic CSDS, including light dark (LDT), EPM, sucrose preference test (SPT), and NSF (Fig. 2a). A $2 \times 3$ ANOVA (sex $x$ stress susceptibility) revealed no main effect of sex within LDT (light time $p=0.51$; light distance $p=0.37$ ), EPM (open arm time $p=0.61$; percent distance in open arms $p=0.91)$, or sucrose preference $(p=0.91)$. Within the LDT a significant main 
A

CNDS Mice

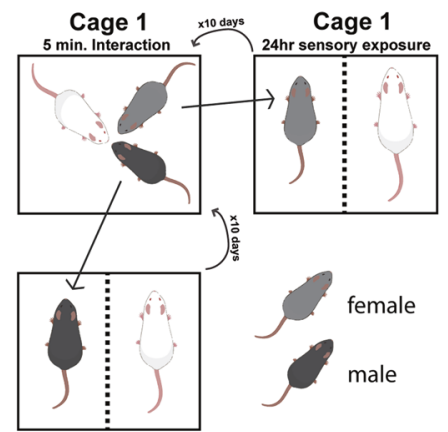

Cage 2

$24 \mathrm{hr}$ sensory exposure

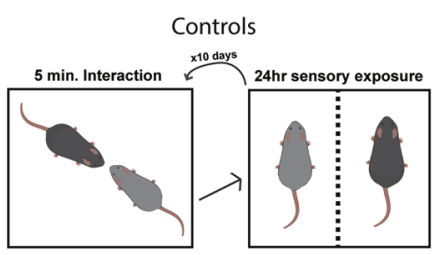

C

C Male

$1.30 \%$
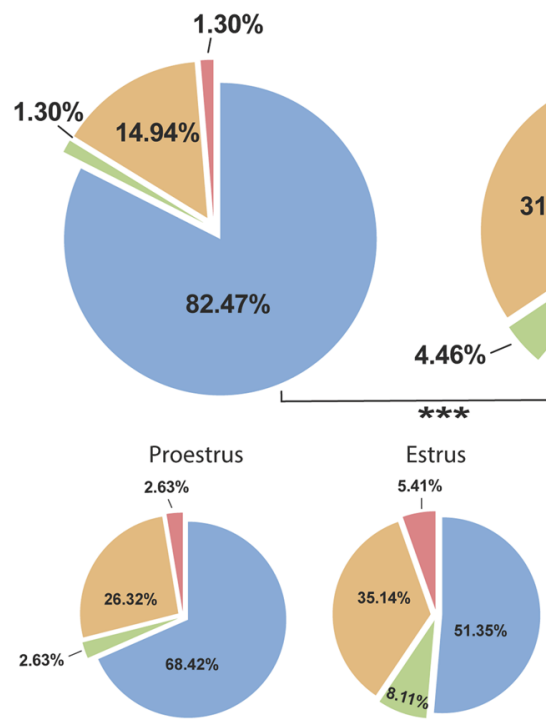

D

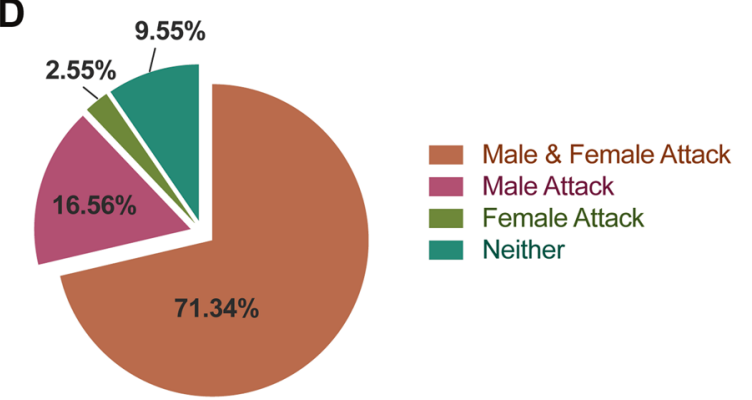

B
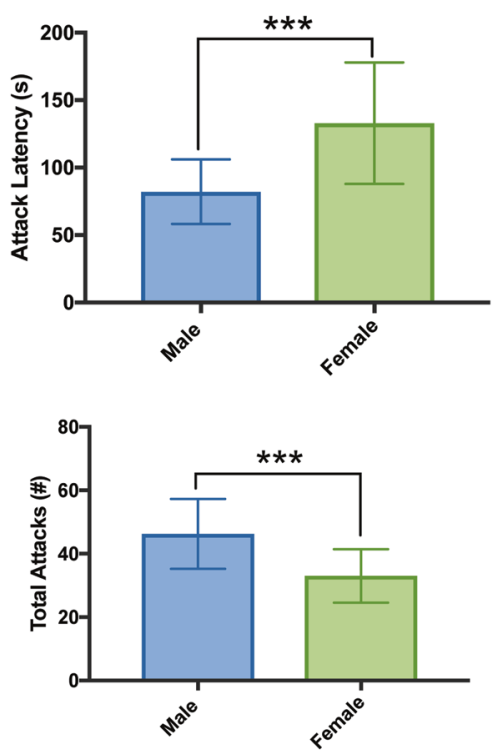

Female $\quad$ Aggression Only

Mounting Only

- Neither

$3.18 \%$

Mounting \& Aggression 
A - CNTRL - RES - SUS

B

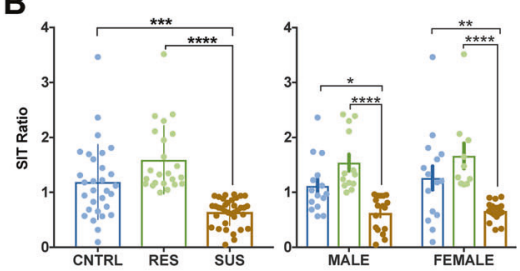

E

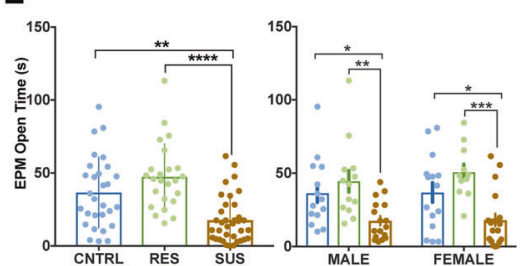

H

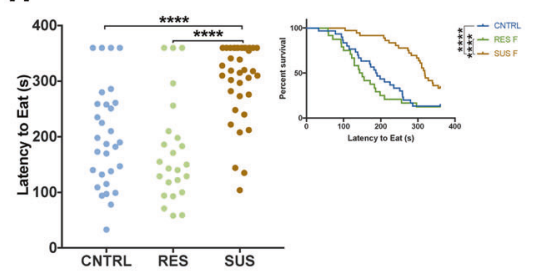

J
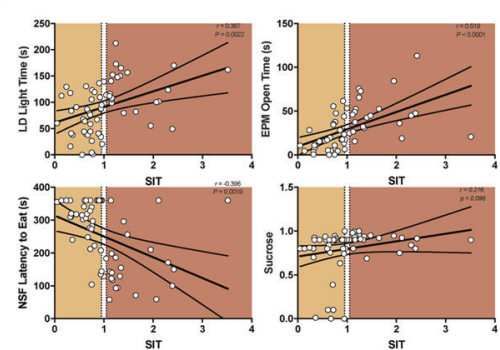
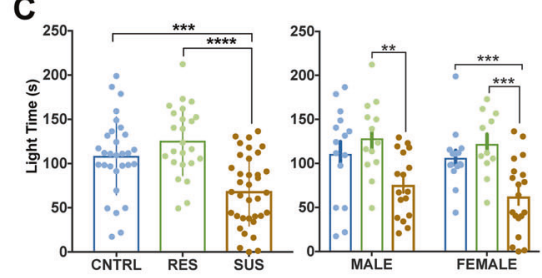

F
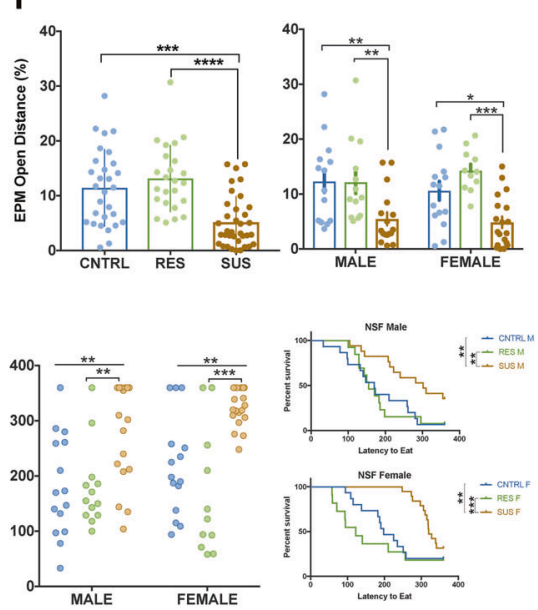

K
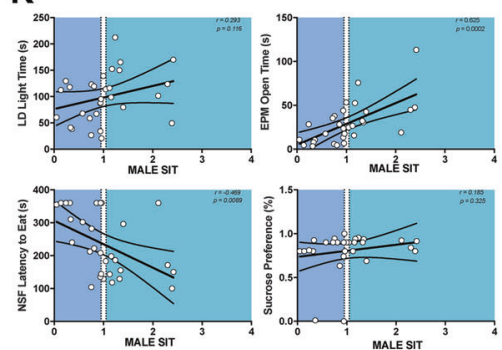

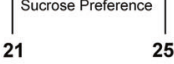

D
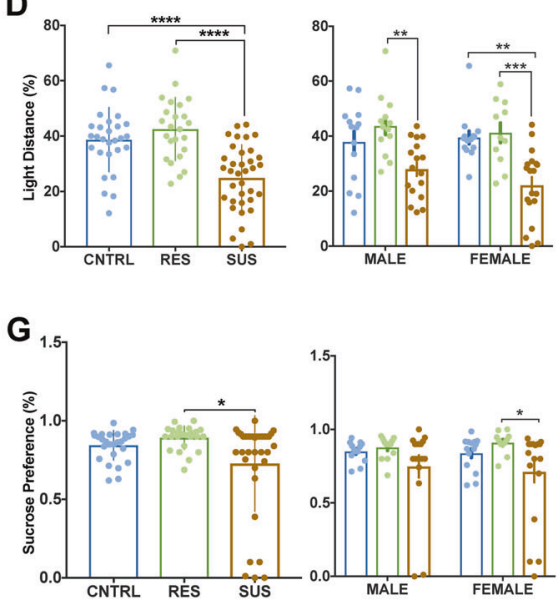

I

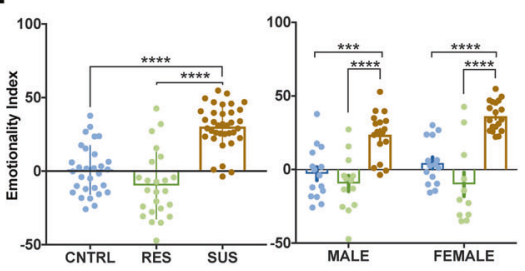

L

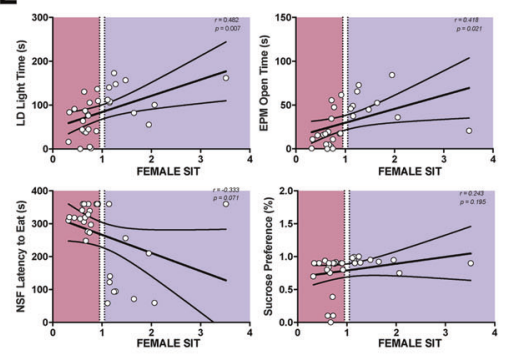

Fig. 2 CNSDS SUS male and female mice have an increase in negative valence behaviors. a Timeline of CNSDS paradigm and behavior. b-g represent separate $2 \times 3$ ANOVAs with the left graph representing collapsed groups to explore the main effect of stress susceptibility phenotype, while the right displays planned Bonferroni post-hoc comparisons (SEX $\times$ STRESS). Main effect of stress was observed in SIT $\left(F(2,83)=23.21, p<0.001 ; * * * *\right.$ SUSvSRES $p<0.0001 ; * * *$ SUSvsCNTRL $p=0.0003$; male ****SUSvsRES $p<0.0001$; ${ }^{*}$ SUSvsCNTRL $p=0.036$; female $* * *$ SUSvsRES $p<0.0001 ; * *$ SUSvsCNTRL $p=0.005)$, LDT light time $(F(2,84)=16.64, p<0.001 ; * * *$ SUSvsRES $p<0.0001 ; * *$ SUSvsCNTRL $p=$ 0.0006; male **SUSvsRES $p=0.002$; female ${ }^{* * *}$ SUSvsRES $p<0.001$; ${ }^{* * *}$ SUSvSCNTRL $\left.p<0.001\right)$, LDT light distance $(F(2,84)=14.79, p<0.001$;

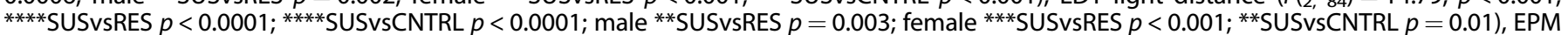
open arm time $\left(F(2,83)=15.33, p<0.001 ; * * * *\right.$ SUSvsRES $p<0.0001 ; * *$ SUSVSCNTRL $p=0.0018$; male **SUSVsRES $p=0.003$; ${ }^{*}$ SUSVSCNTRL $p=$ 0.047 ; female $* * *$ SUSVSRES $p<0.001 ;{ }^{*}$ SUSVSCNTRL $\left.p=0.035\right)$, EPM open arm distance $\left(F(2,83)=15.7, p<0.001\right.$; ${ }^{* * * *}$ SUSVSRES $p<0.0001$; ${ }^{* * *}$ SUSvsCNTRL $p=0.0002$; male **SUSvsRES $p=0.004 ; * *$ SUSVsCNTRL $p=0.015$; female ***SUSvsRES $p<0.001 ;{ }^{*}$ SUSvSCNTRL $p=0.045$ ), in sucrose preference $(F(2,80)=4.79, p=0.011$; *SUSvSRES $p=0.0125$; females *SUSvsRES $p=0.044)$. Scatterplots $\mathbf{h}$ represents feeding behavior differences from Bonferroni Kaplan-Meier survival analysis between the stress susceptibility phenotypes ${ }^{* * *}$ SUSvsRES $\left(X^{2}(1) 17.75, p=\right.$ $0.0035) * * *$ SUSvsCNTRL $\left(X^{2}(1)=14.94, p=0.0065\right)$. Separate Bonferroni Kaplan-Meier survival analysis were also conducted between sexes with male ${ }^{*}$ SUSvsRES $\left(X^{2}(1) 8.51, p=0.0035\right),{ }^{*} \operatorname{SUSVSCNTRL}\left(X^{2}(1)=7.4, p=0.0065\right)$ and female ${ }^{* *}$ SUSvsRES $\left(X^{2}(1) 6.81, p=0.0009\right)$, ${ }^{*}$ SUSvSCNTRL $\left(X^{2}(1)=6.4, p=0.01\right)$. i represents separate $2 \times 3$ ANOVAs with the left graph representing collapsed groups to explore the main effect of stress susceptibility phenotype, while the right displays planned Bonferroni post-hoc comparisons (SEXxSTRESS) for emotionality index $\left(F_{(2,84)}=43.34, p<0.0001, * * * *\right.$ SUSvSRES $p<0.0001 ; * * * *$ SUSVSCNTRL $p<0.0001 ;$ male ****SUSvSRES $p<0.0001$; ${ }^{* * *}$ SUSvsCNTRL $p=0.0001$; female ***SUSvSRES $p<0.0001 ; * * *$ SUSvSCNTRL $p<0.0001$. j Correlations collapsed by sex showed significant relationships between SIT ratios and time spent in the light, in EPM open arms, and NSF latency to eat. $\mathbf{k}$ Correlations within males revealed significant relationships between SIT ratios and time spent on the EPM open arms as well as NSF latency to eat. I In females, significant correlations emerged between SIT ratios and time spent light, and on the EPM open arms

effect of stress was observed in time spent $(F(2,84)=16.64, p<$ $0.001)$ and distance traveled $(F(2,84)=14.79, p<0.001)$ in the light, with SUS mice spending less time (RES: $p<0.0001$; CNTRL: $p=$ 0.0006; Fig. $2 c$ left) and traveling less distance (RES: $p<0.0001$;
CNTRL: $p<0.0001$; Fig. $2 \mathrm{~d}$ left) than RES and CNTRL mice. Planned Bonferroni post-hoc comparisons revealed SUS males spent less time ( $p=0.002$; Fig. $2 c$ right) and traveled less in the light ( $p=$ 0.003; Fig. 2d right) than RES males, while SUS females spent less 
time (RES: $p<0.001$; CNTRL: $p<0.001$; Fig. $2 c$ right) and traveled less (RES: $p<0.001$; CNTRL: $p=0.01$; Fig. $2 \mathrm{~d}$ right) in the light than RES and CNTRL females. Significant effects of stress were also found in time spent $(F(2,83)=15.33, p<0.001)$ and percent distance traveled $(F(2,83)=15.7, p<0.001)$ in EPM open arms, with SUS mice spending less time (RES: $p<0.0001$; CNTRL: $p=0.0018$; Fig. 2e left) and traveling less on the EPM open arms (RES: $p<$ 0.0001 ; CNTRL: $p=0.0002$; Fig. $2 \mathrm{f}$ left) than RES and CNTRL mice. Planned Bonferroni post-hoc comparisons indicated that SUS males spent less time (RES: $p=0.003$; CNTRL $p=0.047$; Fig. 2e) and traveled less (RES: $p=0.004$; CNTRL $p=0.015$; Fig. $2 f$ ) in EPM open arms than RES and CNTRL males and that SUS females also spent less time (RES: $p<0.001$; CNTRL $p=0.035$; Fig. 2e right) and traveled less (RES: $p<0.001$; CNTRL $p=0.045$; Fig. 2f right) than RES and CNTRL females in the EPM open arms. There was also a significant effect of stress $(F(2,80)=4.79, p=0.011$; Fig. $2 \mathrm{~g})$ in sucrose consumption, with SUS mice having a lower preference for $1 \%$ sucrose solution than RES mice ( $p=0.0125$; Fig. $2 \mathrm{~g}$ left). Planned Bonferroni post-hoc comparisons revealed that this effect was observed in females, with SUS females showing less preference for a $1 \%$ sucrose solution than RES females ( $p=$ 0.044; Fig. $2 \mathrm{~g}$ right). Lastly, in the NSF, Kaplan-Meier survival analyses between the stress susceptibility phenotypes showed a significant effect of stress susceptibility phenotype $\left(X^{2}(2)=20.53\right.$, $p<0.0001)$, with SUS mice having a longer latency to eat than RES $\left(X^{2}(1)=17.75, p=0.0035\right)$ and CNTRL $\left(X^{2}(1)=14.94, p=0.0065\right)$ mice (Fig. $2 \mathrm{~h}$ left) with both sexes collapsed. We also examined the effects of CNSDS on NSF within each sex. SUS males had a longer latency to eat than RES $\left(X^{2}(1)=8.51, p=0.0035\right.$; Bonferroni corrected) and CNTRL $\left(X^{2}(1)=7.4, p=0.0065\right.$; Bonferroni corrected) males (Fig. $2 \mathrm{~h}$ right) and SUS females had a longer latency to eat than $\operatorname{RES}\left(X^{2}(1)=6.81, p=0.0009\right.$; Bonferroni corrected) and CNTRL $\left(X^{2}(1)=6.4, p=0.01\right.$; Bonferroni corrected) females (Fig. $2 \mathrm{~h}$ right). No differences in home cage feeding behavior were observed (Supplemental Fig. 3C).

Next, we used an emotionality index to assess CNSDS-induced behavioral differences across LD, EPM, Sucrose Preference, and NSF. As previously described [32], this emotionality index calculates $z$-scores for each behavioral test (LD, EPM, Sucrose, and NSF) by normalizing individual mice against CNTRL averages and the standard deviation for that specific behavior. This allows a comprehensive analysis of multiple behavioral modalities. A score above zero represents a mouse with less time and distance in the light of the LD, less time and distance on EPM open arms, low preference for $1 \%$ sucrose solution, and longer latency to feed in the NSF task relative to CNTRL. A $2 \times 3$ ANOVA showed that there was a significant effect of stress susceptibility phenotype $\left(F_{(2,84)}=\right.$ $43.34, p<0.0001)$, but no main effect of sex $(p=0.085)$. Bonferroni post-hoc comparisons showed that SUS mice have a higher emotionality index than RES $(p<0.0001)$ and CNTRL $(p<0.0001)$ with all mice collapsed (Fig. 2i left). Planned Bonferroni post-hoc comparisons within sex showed that SUS males have a

A

\begin{tabular}{|c|c|c|c|c|c|c|c|}
\hline PROESTRUS & $\begin{array}{l}\text { Chronic Non-Discriminatory } \\
\text { Social Defeat Stress }\end{array}$ & SIT & OFT & LDT & EPM & NSF & Sucrose Preference \\
\hline METESTRUS & 1 & 11 & 13 & 15 & 17 & 19 & 21 \\
\hline
\end{tabular}

B

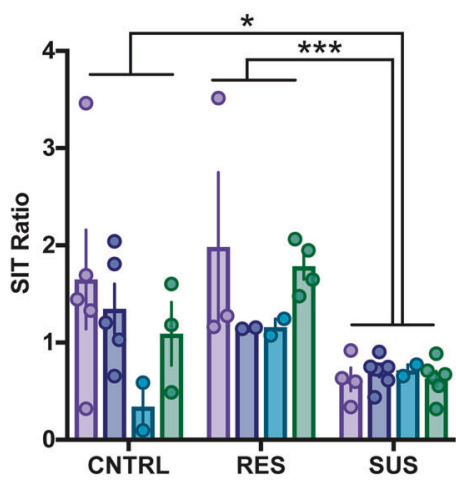

D

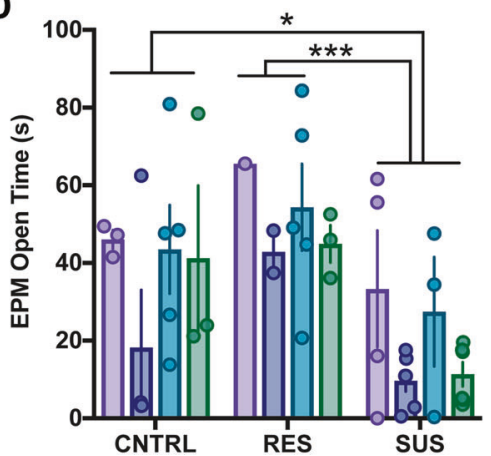

C

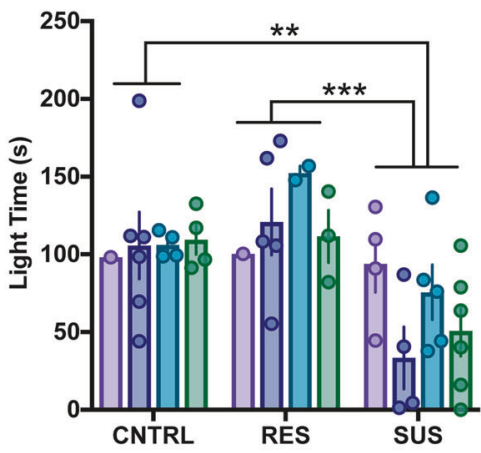

E

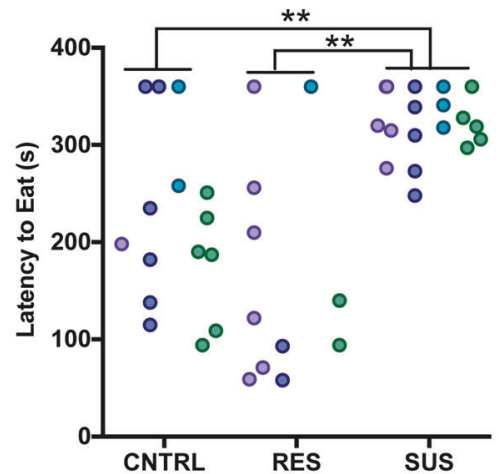

Fig. 3 Female negative valence behavior is not impacted by estrous cycle stage during behavioral testing. $\mathbf{a}$ Time line of experiment. $\mathbf{b}-\mathbf{d} 3 \times 4$ ANOVAs revealed that stress susceptibility phenotype effects persisted in the SIT $\left(F(3,33)=6.44, p=0.004 ;{ }^{* *}\right.$ SUS vs. RES $p<0.001 ;{ }^{*}$ SUS vs CNTRL $p=0.018)$, LDT $\left(F(3,33)=7.62, p=0.002 ;{ }^{* *}\right.$ SUS vs. RES $p<0.001 ;{ }^{*}$ SUS vs CNTRL $p=0.007$ Bonferroni corrected $)$, and EPM $(F(3,33)=$ $6.65, p=0.004 ;{ }^{* *}$ SUS vs. RES $p<0.001 ;{ }^{*}$ SUS vs CNTRL $p=0.041$ Bonferroni corrected). e Kaplan-Meier analysis also revealed differences in NSF latency to eat $\left(X^{2}(11)=57.69, p<0.001 ;{ }^{* *}\right.$ SUS vs. RES $p=0.01 ;{ }^{* *}$ SUS vs CNTRL $p=0.01$ Bonferroni corrected) 
higher emotionality index score than CNTRL $(p=0.0001)$ and RES $(p<0.0001)$ males (Fig. 2i right). Similarly, SUS females had a higher emotionality index score than CNTRL $(p<0.0001)$ and RES $(p<0.0001)$ females (Fig. 2i right).

To further assess the relationship between stress susceptibility phenotype and the other behaviors, separate correlations revealed that SIT ratio is significantly related to LD light time $(r=0.387, p=0.0022$; Fig. 2j), EPM open arm time $(r=0.519, p<$ 0.0001 ; Fig. 2j), and NSF latency to eat $(r=-0.393, p=0.0019$; Fig. 2j) when both sexes were collapsed. There was not a significant relationship between SIT ratio and sucrose preference $(r=0.216, p=0.096)$. We also examined the effects of CNSDS on these behavior correlations within each sex. SIT ratio in males was related to EPM open arm time ( $r=0.625, p=0.0002$; Fig. $2 \mathrm{k})$, and NSF latency to eat ( $r=-0.496, p=0.0089$; Fig. $2 \mathrm{k}$ ). In females, SIT ratio was significantly related to LD light time $(r=0.482, p=$ 0.007; Fig. 2l) and EPM open arm time $(r=0.418, p=0.021$; Fig. 2l).

We also ran an additional control group in which three mice interacted (two males and one female; 2-male CNTRL) and compared the effects on behavior to what was observed when two mice interacted (one male and one female; 1-male CNTRL) (Supplemental Fig. 2). We found no differences in any of the behavioral tasks (Supplemental Fig. 2B-I).

Taken together, these results demonstrate that the CNSDS paradigm effectively induces susceptible and resilient behavioral phenotypes in both males and females. Both SUS male and SUS female mice displayed increases in negative valence behaviors relative to RES and CNTRL mice and there were no sex differences in negative valence behaviors or sucrose preference. Thus, the CNSDS paradigm yields comparable stress effects on behavior in both sexes.

Estrous cycle does not impact stress susceptibility phenotype Within females, the behavioral results were not impacted by the estrous cycle since $3 \times 4$ ANOVAs (stress susceptibility $\times$ estrous cycle phase) revealed no main effect of estrous cycle phase in SIT $(p=0.159)$, LDT $(p=0.472)$, or EPM $(p=0.105)$ (Fig. 3a-d).
Kaplan-Meier analysis also showed no difference in latency to eat in NSF across estrous ( $p=0.768$; Fig. 3e). Furthermore, when analyzing potential effects of the four stages of estrous on behavior, the main effect of stress susceptibility persisted in SIT ratio $(F(3,33)=6.44, p=0.004$; SUS vs. RES $p<0.001$; SUS vs CNTRL $p=0.018$ Bonferroni corrected), LDT time in light $(F(3,33)=7.62$, $p=0.002$; SUS vs. RES $p<0.001$; SUS vs CNTRL $p=0.007$ Bonferroni corrected), and EPM time in open arms $(F(3,33)=$ $6.65, p=0.004$; SUS vs. RES $p<0.001$; SUS vs CNTRL $p=0.041$ Bonferroni corrected; Fig. 3b-d). Kaplan-Meier analysis also revealed stress susceptibility phenotype differences in NSF latency to eat $\left(X^{2}(11)=57.69, p<0.001\right.$; SUS vs. RES $p=0.01$; SUS vs CNTRL $p=0.01$ Bonferroni corrected; Fig. 3e). No significant differences were observed in home cage feeding behavior between estrous phases and stress susceptibility phenotype (Supplemental Fig. 3D). These data indicate that the estrous cycle does not impact stress susceptibility or behavior in stressed and non-stressed freely cycling female C57BL/6J mice. Furthermore, CNSDS did not affect the estrous cycle. CNSDS-exposed and CNTRL females had similar cycle lengths prior to and during either CNSDS or CNTRL exposure (Supplemental Fig. 3A). RES and SUS females also had similar cycle lengths both prior to and during CNSDS exposure (Supplemental Fig. 3B).

CNSDS increases plasma corticosterone levels

Increases in HPA axis activation are found in response to CSDS stress [33-35]. Therefore, we next evaluated whether the CNSDS paradigm elicited increases in plasma corticosterone levels, with blood collected before CNSDS (baseline) and again on the final day of CNSDS exposure, following the last 5-min interaction (defeat; Fig. 4a). To analyze differences between sex and stress susceptibility phenotype (RES, SUS, and CNTRL) across the two blood collection time points (baseline, defeat) a repeated measures $2 \times 2 \times 3$ ANOVA revealed significant main effects of stress $(F(2,60)=15.6, p<0.0001)$ and time point $F(1,60)=17.17$, $p=0.0001)$, with a significant interaction between stress susceptibility phenotype and blood collection time point $(F(2,60)=10.86$, $p<0.0001)$. There were no significant effects of sex $(p=0.215)$ on

A

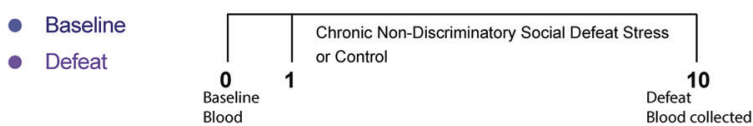

B

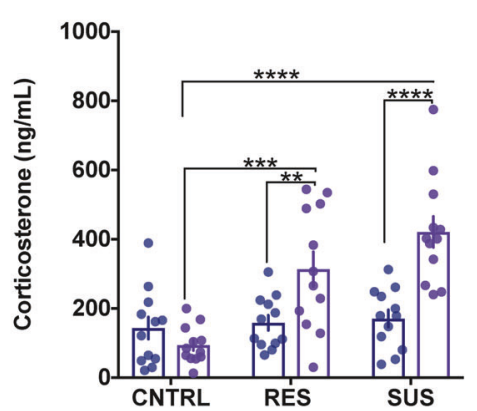

C

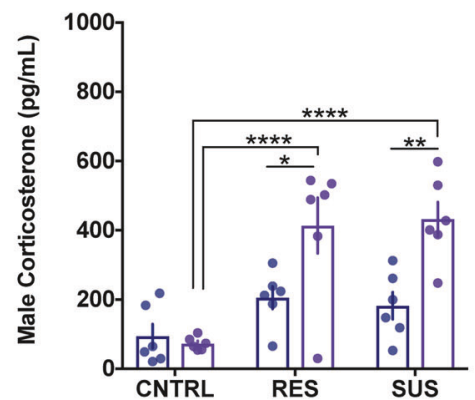

D

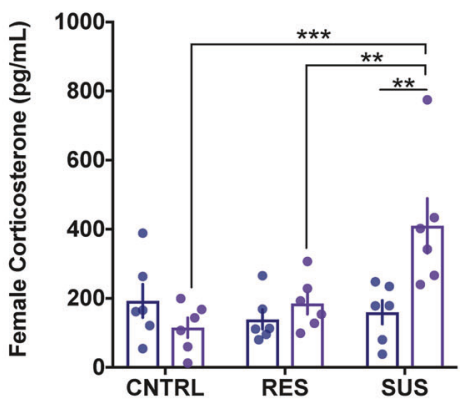

Fig. 4 CNSDS increases plasma corticosterone levels. a Timeline of retro-orbital eye bleeds. $\mathbf{b}-\mathbf{d} 2 \times 2 \times 3$ repeated measures ANOVA revealed significant main effects of stress susceptibility phenotype $(F(2,60)=15.6, p<0.0001)$, time point $F(1,60)=17.17, p=0.0001)$, and interaction between stress susceptibility phenotype and blood collection time point $(F(2,60)=10.86, p<0.0001)$. Bonferroni post-hoc comparisons collapsed by sex (b) showed significant differences in corticosterone response following CNSDS exposure $(* * * * S U S$ vs CNTRL $p<0.0001$; ***RES vs CNTRL $p=0.0001)$. CNSDS also increased corticosterone levels relative to baseline in both RES $(* * p=0.0059)$ and SUS $(* * * *<<$ 0.0001). In males (c) Bonferroni post-hoc comparisons showed differences in corticosterone in response to CNSDS exposure (****SUS vs CNTRL $p<0.0001$ and ${ }^{* * * *}$ RES vs CNTRL $p<0.0001$ ). Additionally, CNSDS increased corticosterone levels relative to baseline in both SUS (** $p=$ $0.0018)$ and RES ( $\left.{ }^{*} p=0.0098\right)$ males. d Bonferroni post-hoc comparisons in females showed differences in corticosterone in response to CNSDS exposure ${ }^{* *}$ SUS vs CNTRL $p=0.0002$ and ${ }^{* *}$ SUS vs RES $\left.p=0.0043\right)$. Lastly, CNSDS significantly increased corticosterone levels between baseline and defeat only in SUS females $\left({ }^{* *} p=0.0018\right)$ 
plasma corticosterone levels. Bonferroni post-hoc comparisons demonstrated that CNTRL mice had lower corticosterone levels than RES $(p=0.0001)$ and SUS $(p<0.0001)$ following exposure to CNSDS (Fig. 4b) when all mice were collapsed. Additionally, corticosterone levels following CNSDS interaction were significantly different relative to baseline in both SUS $(p<0.0001)$ and RES ( $p=0.0059$; Fig. $4 b)$. We next ran planned Bonferroni post-hoc comparisons within each sex. In males, CNTRL mice had lower corticosterone levels than SUS $(p<0.0001)$ and RES $(p<0.0001)$ males following the final CNSDS interaction (Fig. 4c), and CNSDS increased corticosterone levels relative to baseline in both SUS $(p=0.0018)$ and RES $(p=0.0098)$ males (Fig. $4 c)$. In females, SUS mice had higher corticosterone plasma levels than RES ( $p=$ 0.0043 ) and CNTRL ( $p=0.0002)$ females following the final CNSDS interaction (Fig. 4d), and CNSDS significantly increased corticosterone levels between baseline and defeat only in SUS females ( $p$ $=0.0018$; Fig. 4d). RES females subjected to CNSDS did not show an increase in corticosterone levels relative to their baseline ( $p=$ 0.99). Taken together, these data indicate that CNSDS results in a significant increase in HPA axis activation in both SUS males and SUS females as well as in RES males.

\section{DISCUSSION}

Several chronic stress paradigms that are used in rodents are ineffective in females. One such example is chronic social defeat stress. Recently described modifications that involve chemogenetics or application of male urine to females have permitted usage of social defeat in females. However, these clever modifications require difficult surgeries or tedious urine collection $[24,25]$. Here we develop a streamlined novel non-discriminatory social defeat paradigm (CNSDS), that, while similar to CSDS, induces chronic stress behavioral and neuroendocrine phenotypes in both males and females of the widely-used C57BL6/J strain. CNSDS has face validity as it permits stratification of stressed male and female mice into SUS and RES groups. This stratification is similar to the stress response observed in males in classic CSDS $[27,31]$ and in females in modified CSDS paradigms [24, 25]. Therefore, the CNSDS paradigm successfully induces chronic stress behavioral phenotypes in both males and females.

To the best of our knowledge, the only other chronic stressor that simultaneously exposes males and females to stress is the vicarious social defeat paradigm $[36,37]$. Within this paradigm, male C57BL/6J mice undergo physical defeat stress, while female mice experience emotional stress through exposure (separated by a divider) to the aggressive bouts displayed from CD-1 aggressors to C57BL/6J mice. However, unlike CNSDS, in which the same physical stressor is applied to both sexes, comparison between the sexes in vicarious social defeat is difficult because the males experience physical stress and females experience emotional stress. Although female-to-female aggression occurs infrequently, a recent study showed that housing intact swiss webster (CFW) female mice with a castrated male resulted in CFW females displaying aggressive behavior to intruding C57BL/6J female mice [38]. These defeated females displayed reduced social interaction, which was then reversed by ketamine treatment. This paradigm is very interesting, but one advantage that CNSDS offers is that it permits some direct comparisons of chronic stress responses between sexes, as both males and females are being exposed to the same aggressor and experience simultaneously.

Male-to-female aggression is rare in classic CSDS, as resident CD-1 males are more likely to display mounting behaviors against female intruders [20]. However, CD-1 males attack male intruders $[27,39,40]$. In CNSDS, CD-1 aggressors attack both males and females possibly because of a generalized aggressive response to the male intruders. Although the CD-1 aggressors attacked males more frequently and with a shorter latency than females, the attacks of the females were sufficient to induce RES and SUS behavioral and neuroendocrine phenotypes similar to what was observed in males. Thus, similar to what is observed when comparing SUS and RES mice in traditional CSDS [18], the number of attacks and latencies to attack do not impact stress susceptibility phenotypes when comparing males and females.

Overall the CNSDS paradigm affected both sexes similarly in the LDT, EPM, NSF, and SPT. SUS male and female mice displayed increases in negative valence behaviors associated with anxiety relative to RES and CNTRL same sex counterparts. However, CNSDS also resulted in a significantly increased sucrose preference in RES females relative to SUS females, a finding that was not observed in males. While classic CSDS is often reported to result in differences in sucrose preference between SUS and RES males, some studies report more mixed results [41, 42]. Furthermore, we found no effect of C57BL/6J estrous stage on CD-1 attack behavior of females in the CNSDS paradigm, which could be due to the presence of $\mathrm{C} 57 \mathrm{BL} / 6 \mathrm{~J}$ male mice during all defeat interactions $[43,44]$. Similarly, Harris and colleagues [25] also found that estrous did not impact CD-1 attack behavior in their modified version of CSDS that applied urine to C57BL/6J females. Thus, CNSDS permits direct comparison of chronic stress effects on males and females without the need for estrous cycle tracking.

Previous studies have demonstrated that female mice spend less time in the open arms than male equivalents during diestrus, but spend more time in the open arms during proestrus [45, 46]. Studies that collapse all rodents regardless of estrous phase have found that female rats spend more time in the open arms than males [47]. Previous studies also found baseline sex differences in sucrose preference, with female rats and mice consuming more sucrose than males $[48,49]$. The lack of baseline sex differences in this study may be due to strain, as other studies have also observed no sex differences in C57BL6 mice at baseline [50, 51].

To assess the effects of CNSDS on the HPA axis, blood samples were collected prior to CNSDS and again on the last day of CNSDS. Overall, both SUS male and female mice showed an increase in corticosterone levels after 10 days of defeat relative to baseline levels before stress exposure and compared to CNTRL. Similarly, classic CSDS increases plasma corticosterone levels in male experimental mice [34], and modified CSDS increases plasma corticosterone levels in female experimental mice [25]. However, within males we found that CNSDS increased corticosterone levels in both RES and SUS mice, which is similar to the findings of Krishnan and colleagues [18] using the traditional CSDS paradigm. By contrast, SUS females displayed higher corticosterone levels than RES females following CNSDS. These data suggest that CNSDS induces a robust corticosterone response specifically in SUS females.

While clever modifications of the classic CSDS protocol exist for inducing stress in female mice, CNSDS is a streamlined paradigm that does not require difficult, time-consuming surgeries or urine collection [24, 25] and doubles experimental output by allowing the simultaneous defeat of two mice (one male and one female). However, one potential drawback to CNSDS is that experimental C57BL6/J males and females receive different levels of attack from CD-1 aggressors. While CNSDS does induce similar behavioral effects and stress susceptibility phenotypes in males and females, the attack differences may be a confound. One advantage of the Takahashi and colleagues paradigm [24] is that different levels of CD-1 aggression can be achieved through chemogenetic approaches.

Overall, the CNSDS paradigm leads to similar behavioral and neuroendocrine effects in both male and female C57BL/6J mice. Adoption of this streamlined CNSDS paradigm will help advance the initiative to include female rodents in preclinical chronic stress research. Thus, we present a CNSDS paradigm to simultaneously investigate the mechanisms underlying stress susceptibility and resilience in both sexes as well as the potential to study the neural circuitry underlying therapeutic interventions in both sexes. 


\section{FUNDING AND DISCLOSURE}

The authors declare no competing interests. This work was funded by NIMH Grant R01 MH112861 (BAS). The authors declare no competing interests.

\section{ACKNOWLEDGEMENTS}

We would like to thank Taylor Anthony, Sandra Ashamalla, Leshya Bokka, and Debbie Ma for assistance in data collection.

\section{ADDITIONAL INFORMATION}

Supplementary Information accompanies this paper at (https://doi.org/10.1038/ s41386-019-0520-7).

Publisher's note Springer Nature remains neutral with regard to jurisdictional claims in published maps and institutional affiliations.

\section{REFERENCES}

1. Kessler RC, McGonagle KA, Zhao S, Nelson CB, Hughes M, Eshleman S, et al. Lifetime and 12-month prevalence of DSM-III-R psychiatric disorders in the United States. Results from the National Comorbidity Survey. Arch Gen Psychiatry. 1994;51:8-19.

2. Beery AK, Zucker I. Sex bias in neuroscience and biomedical research. Neurosci Biobehav Rev. 2011;35:565-72.

3. Becker JB, Arnold AP, Berkley KJ, Blaustein JD, Eckel LA, Hampson E, et al. Strategies and methods for research on sex differences in brain and behavior. Endocrinology. 2005;146:1650-73.

4. Shansky RM. Sex differences in PTSD resilience and susceptibility: challenges for animal models of fear learning. Neurobiol Stress. 2015;1:60-65.

5. Yohn CN, Shifman S, Garino A, Diethorn E, Bokka L, Ashamalla SA, et al. Fluoxetine effects on behavior and adult hippocampal neurogenesis in female C57BL/6J mice across the estrous cycle. bioRxiv. 2018:368449.

6. Becker JB, Prendergast BJ, Liang JW. Female rats are not more variable than male rats: a meta-analysis of neuroscience studies. Biol Sex Differ. 2016;7:34.

7. Prendergast BJ, Onishi KG, Zucker I. Female mice liberated for inclusion in neuroscience and biomedical research. Neurosci Biobehav Rev. 2014;40:1-5.

8. Shansky RM. Are hormones a "female problem" for animal research? Science. 2019;364:825-26.

9. Berton O, Aguerre S, Sarrieau A, Mormede P, Chaouloff F. Differential effects of social stress on central serotonergic activity and emotional reactivity in Lewis and spontaneously hypertensive rats. Neuroscience. 1998;82:147-59.

10. Calvo N, Cecchi M, Kabbaj M, Watson SJ, Akil H. Differential effects of social defeat in rats with high and low locomotor response to novelty. Neuroscience. 2011;183:81-9.

11. David DJ, Samuels BA, Rainer O, Wang JW, Marsteller D, Mendez I, et al. Neurogenesis-dependent and -independent effects of fluoxetine in an animal model of anxiety/depression. Neuron. 2009;62:479-93.

12. Haller J, Fuchs E, Halasz J, Makara GB. Defeat is a major stressor in males while social instability is stressful mainly in females: towards the development of a social stress model in female rats. Brain Res Bull. 1999;50:33-9.

13. Hodes GE, Pfau ML, Purushothaman I, Ahn HF, Golden SA, Christoffel DJ, et al. Sex Differences in nucleus accumbens transcriptome profiles associated with susceptibility versus resilience to subchronic variable stress. J Neurosci. 2015;35:16362-76.

14. Meerlo P, Overkamp GJ, Daan S, Van Den Hoofdakker RH, Koolhaas JM. Changes in behaviour and body weight following a single or double social defeat in rats. Stress. 1996:1:21-32.

15. Trainor BC, Pride MC, Villalon Landeros R, Knoblauch NW, Takahashi EY, Silva AL, et al. Sex differences in social interaction behavior following social defeat stress in the monogamous California mouse (Peromyscus californicus). PLoS ONE. 2011;6:e17405.

16. Koolhaas JM, De Boer SF, De Rutter AJ, Meerlo P, Sgoifo A. Social stress in rats and mice. Acta Physiol Scand Suppl. 1997;640:69-72.

17. Chaudhury D, Walsh JJ, Friedman AK, Juarez B, Ku SM, Koo JW, et al. Rapid regulation of depression-related behaviours by control of midbrain dopamine neurons. Nature. 2013:493:532-6.

18. Krishnan V, Han MH, Graham DL, Berton O, Renthal W, Russo SJ, et al. Molecular adaptations underlying susceptibility and resistance to social defeat in brain reward regions. Cell. 2007;131:391-404.

19. Larrieu T, Cherix A, Duque A, Rodrigues J, Lei H, Gruetter R, et al. Hierarchical status predicts behavioral vulnerability and nucleus accumbens metabolic profile following chronic social defeat stress. Curr Biol. 2017;27:2202-10 e4.
20. DeBold JF, Miczek KA. Aggression persists after ovariectomy in female rats. Horm Behav. 1984;18:177-90.

21. Smith AS, Lieberwirth C, Wang Z. Behavioral and physiological responses of female prairie voles (Microtus ochrogaster) to various stressful conditions. Stress. 2013;16:531-9.

22. Bosch OJ, Neumann ID. Vasopressin released within the central amygdala promotes maternal aggression. Eur J Neurosci. 2010;31:883-91.

23. Bourke $\mathrm{CH}$, Neigh GN. Exposure to repeated maternal aggression induces depressive-like behavior and increases startle in adult female rats. Behav Brain Res. 2012;227:270-5.

24. Takahashi A, Chung JR, Zhang S, Zhang H, Grossman Y, Aleyasin H, et al. Establishment of a repeated social defeat stress model in female mice. Sci Rep. 2017;7:12838.

25. Harris AZ, Atsak P, Bretton ZH, Holt ES, Alam R, Morton MP, et al. A novel method for chronic social defeat stress in female mice. Neuropsychopharmacology. 2018;43:1276-83.

26. Tsankova NM, Berton O, Renthal W, Kumar A, Neve RL, Nestler EJ. Sustained hippocampal chromatin regulation in a mouse model of depression and antidepressant action. Nat Neurosci. 2006;9:519-25.

27. Golden SA, Covington HE III, Berton O, Russo SJ. A standardized protocol for repeated social defeat stress in mice. Nat Protoc. 2011;6:1183-91.

28. Byers SL, Wiles MV, Dunn SL, Taft RA. Mouse estrous cycle identification tool and images. PLoS ONE. 2012;7:e35538.

29. McLean AC, Valenzuela N, Fai S, Bennett SA. Performing vaginal lavage, crystal violet staining, and vaginal cytological evaluation for mouse estrous cycle staging identification. J Vis Exp. 2012;15:e4389.

30. Felicio LS, Nelson JF, Finch CE. Longitudinal studies of estrous cyclicity in aging C57BL/6J mice: II. Cessation of cyclicity and the duration of persistent vaginal cornification. Biol Reprod. 1984;31:446-53.

31. Razzoli M, Carboni L, Andreoli M, Ballottari A, Arban R. Different susceptibility to social defeat stress of BalbC and C57BL6/J mice. Behav Brain Res. 2011;216:100-8.

32. Guilloux JP, Seney M, Edgar N, Sibille E. Integrated behavioral z-scoring increases the sensitivity and reliability of behavioral phenotyping in mice: relevance to emotionality and sex. J Neurosci Methods. 2011;197:21-31.

33. Hammels C, Prickaerts J, Kenis G, Vanmierlo T, Fischer M, Steinbusch HW, et al. Differential susceptibility to chronic social defeat stress relates to the number of Dnmt3a-immunoreactive neurons in the hippocampal dentate gyrus. Psychoneuroendocrinology. 2015;51:547-56.

34. Keeney A, Jessop DS, Harbuz MS, Marsden CA, Hogg S, Blackburn-Munro RE. Differential effects of acute and chronic social defeat stress on hypothalamicpituitary-adrenal axis function and hippocampal serotonin release in mice. J Neuroendocrinol. 2006;18:330-8.

35. Keeney AJ, Hogg S, Marsden CA. Alterations in core body temperature, locomotor activity, and corticosterone following acute and repeated social defeat of male NMRI mice. Physiol Behav. 2001;74:177-84.

36. Iniguez SD, Flores-Ramirez FJ, Riggs LM, Alipio JB, Garcia-Carachure I, Hernandez $M A$, et al. Vicarious social defeat stress induces depression-related outcomes in female mice. Biol Psychiatry. 2018;83:9-17.

37. Sial OK, Warren BL, Alcantara LF, Parise EM, Bolanos-Guzman CA. Vicarious social defeat stress: bridging the gap between physical and emotional stress. J Neurosci Methods. 2016:258:94-103.

38. Newman EL, Covington HE III, Suh J, Bicakci MB, Ressler KJ, DeBold JF, et al. Fighting females: neural and behavioral consequences of social defeat stress in female mice. Biol Psychiatry. 2019;13:pii: S0006-3223(19)31337-X

39. Miczek KA, Maxson SC, Fish EW, Faccidomo S. Aggressive behavioral phenotypes in mice. Behav Brain Res. 2001;125:167-81.

40. Van Loo PL, Van Zutphen LF, Baumans V. Male management: coping with aggression problems in male laboratory mice. Lab Anim. 2003;37:300-13.

41. Covington HE III, Maze I, Vialou V, Nestler EJ. Antidepressant action of HDAC inhibition in the prefrontal cortex. Neuroscience. 2015;298:329-35.

42. Croft AP, Brooks SP, Cole J, Little HJ. Social defeat increases alcohol preference of C57BL/10 strain mice; effect prevented by a CCKB antagonist. Psychopharmacology. 2005;183:163-70.

43. Campbell CS, Ryan KD, Schwartz NB. Estrous cycles in the mouse: relative influence of continuous light and the presence of a male. Biol Reprod. 1976;14:292-9.

44. Whitten WK. Modification of the oestrous cycle of the mouse by external stimuli associated with the male; changes in the oestrous cycle determined by vaginal smears. J Endocrinol. 1958;17:307-13.

45. Frye CA, Petralia SM, Rhodes ME. Estrous cycle and sex differences in performance on anxiety tasks coincide with increases in hippocampal progesterone and 3alpha,5alpha-THP. Pharm Biochem Behav. 2000;67:587-96.

46. Walf AA, Frye CA. The use of the elevated plus maze as an assay of anxietyrelated behavior in rodents. Nat Protoc. 2007:2:322-8.

47. Johnston AL, File SE. Sex differences in animal tests of anxiety. Physiol Behav. 1991;49:245-50. 
Chronic non-discriminatory social defeat is an effective chronic stress... CN Yohn et al.

48. Dalla C, Antoniou K, Kokras N, Drossopoulou G, Papathanasiou G, Bekris S, et al. Sex differences in the effects of two stress paradigms on dopaminergic neurotransmission. Physiol Behav. 2008;93:595-605.

49. Hong S, Flashner B, Chiu M, ver Hoeve E, Luz S, Bhatnagar S. Social isolation in adolescence alters behaviors in the forced swim and sucrose preference tests in female but not in male rats. Physiol Behav. 2012;105:269-75.
50. Adamec R, Head D, Blundell J, Burton P, Berton O. Lasting anxiogenic effects of feline predator stress in mice: sex differences in vulnerability to stress and predicting severity of anxiogenic response from the stress experience. Physiol Behav. 2006;88:12-29.

51. O'Leary TP, Gunn RK, Brown RE. What are we measuring when we test strain differences in anxiety in mice? Behav Genet. 2013;43:34-50. 